MODELING, IDENTIFICATION AND CONTROL, 1994, VOL. 15, NO. 3, 179-189

doi:10.4173/mic.1994.3.8

\title{
A perspective on advanced strategies for process control
}

\author{
DALE E. SEBORG + \\ Keywords: Process control, advanced control strategies, survey paper. \\ This paper provides a personal perspective on the current status of advanced process \\ control strategies. First, these strategies are classified according to the degree which \\ they have been used in industry. Then the most prominent methods are discussed \\ critically with emphasis placed on key design issues and unresolved research \\ problems.
}

\section{Introduction}

Before considering advanced process control strategies, it important to acknowledge that the vast majority of industrial control loops ( $90 \%)$ still rely on various forms of the ubiquitous PID controller. Consequently, it can be argued that the substantial capabilities of modern computer control systems are greatly underutilized. However, a compelling counter argument is that many industrial control problems are quite simple and consequently, a conservatively tuned PI or PID controller is a satisfactory solution. (In fact, it is doubtful whether derivative control action is widely used.) Thus there is little incentive to apply more complicated 'advanced' control techniques for this large class of problems. Instead, advanced control strategies should be reserved for difficult control problems where they can provide significant improvements over conventional single loop control. Although the number of difficult problems is usually small compared to the total number of control loops, they typically involve critical process variables which strongly affect key control objectives such as product quality, process operability, and compliance with environmental standards.

The term 'advanced process control' is highly subjective, meaning different things to different people, depending on their background and experience. A not entirely humorous definition is that an advanced control strategy is any technique which a process engineer has not actually used. Table 1 presents an admittedly subjective classification of process control strategies.

The control strategies in Table 1 are grouped into five categories. Category I consists of conventional control strategies that are well known and have been widely used for several decades. The advanced control strategies in Category II are referred to as classical because they have been used in industry for over 20 years and were described in textbooks written by industrial control engineers in the 1960's (e.g. books by Buckley and Shinskey). The process control strategies in Category III have been widely used in industry and are described in current process control textbooks (e.g. Seborg et al. 1989).

Category IV contains both old and new control strategies that have apparently not been widely used in industry, even though successful industrial applications of each of

\footnotetext{
Received June 1994.

$\dagger$ Department of Chemical Engineering, University of California, Santa Barbara, CA 93106, U.S.A.
} 


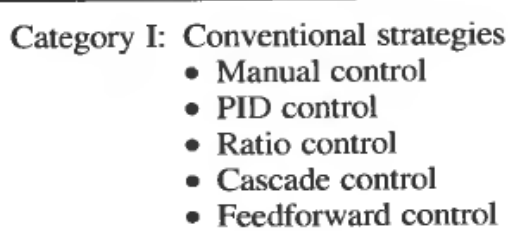

Category II: Advanced control: classical techniques

- Gain scheduling

- Time delay compensation

- Decoupling control

- Selective/override controllers

Category III: Advanced control: widely used techniques

- Model predictive control

- Statistical quality control

- Internal model control

- Adaptive control

Category IV: Advanced control: newer techniques with some industrial applications

- Optimal control (LQG)

- Expert systems

- Nonlinear control

- 'Neurocontrollers'

- Fuzzy control

Category V: Advanced control: proposed strategies with few (if any) industrial applications

Table 1. Classification of process control strategies according to the degree of use in industry.

these methods have been reported. Category $\mathrm{V}$ is a default category that includes new approaches with few, if any, industrial applications. For example, robust control techniques such as $H_{\infty}$ and $\mu$-synthesis are not shown in Table 1 but are considered to be in this category.

The remainder of the paper provides a brief commentary on most of the methods in Categories III and IV. However, internal model control (IMC) is not discussed because it is a general concept, as well as a specific approach, which has been incorporated into many advanced control strategies since it was first published 12 years ago (Garcia and Morari 1982). A second general approach, Linear-Quadratic-Gaussian (LQG) optimal control, is not considered because it is well known and has been available for over 30 years. Although LQG optimal control has not been widely used in the process industries, a number of successful applications have been reported (Fisher and Seborg 1976, Balchen and Mummé 1988). Furthermore, LQG optimal control was an important precursor to the development of the Model Predictive Control (MPC) techniques that are widely used.

In the spirit of previous 'perspectives' on process control (Fisher 1991, Morari 1994), the commentary in this paper must, by necessity, be selective rather than comprehensive. Important related issues such as process identification, process simulation, and control and computing equipment are beyond the scope of this paper.

\section{Multivariable control strategies}

For process control problems with strong interactions between the controlled and manipulated variables, conventional multiloop PID control configurations may not be 
able to provide adequate control. In these situations, the more general multivariable control strategies offer the potential for significant improvements. For the purposes of this paper, the term multivariable control will refer to control strategies in which at least one of the manipulated variables is adjusted based on measured values of more than one controlled variable.

Although multivariable control strategies have been available for over 30 years, widespread application did not take place until the 1980's. For example, a 1976 survey article reported only 29 industrial applications of multivariable control techniques, with 10 of these involving decoupling control systems (Rijnsdorp and Seborg 1976). In a similar vein, a 1980 paper from a leading industrial control group indicated that most of their multivariable control applications involved static decoupling schemes, dynamic decoupling control systems with one way interaction, or full decoupling limited to $2 \times 2$ control problems (Zumwalt and Wolfang 1980).

Next, we discuss two multivariable control strategies that have been widely used in specific industries.

\subsection{Model predictive control}

The most widely used multivariable control strategy is a general approach referred to as model predictive control (MPC). The first MPC techniques were developed independently by two industrial groups in the 1970's. Shell Oil (Houston, TX) reported their 'Dynamic Matrix Control (DMC)' approach in 1979 while a similar technique, IDCOM, was published by a small French company in 1978. Since then, there have been hundreds, if not thousands, of applications of these and related MPC techniques in oil refineries and petrochemical plants around the world. Thus model predictive control has had a substantial impact and is currently the method of choice for difficult control problems in these industries.

One reason why MPC has become a major commercial success is that there are a number of vendors who are licensed to market MPC products and who will install them on a turnkey basis. Consequently, even medium-sized companies are able to take advantage of this new technology. Payout times of 3-12 months are typical (Muske et al. 1991, Gusciora et al. 1992, Richalet 1993).

The basis concept in MPC is that a dynamic model of the process and available measurements are used to predict future process behaviour. The control calculations minimize the difference between the predicted process response and the desired response (Garcia et al. 1989, Fisher 1991, Ricker 1991, Richalet 1993). The MPC control strategy was developed for difficult multi-input, multi-output control (MIMO) problems where there are significant interactions between the manipulated inputs and the controlled outputs. A key advantage of MPC is that it can accommodate inequality constraints on the various process variables. An adaptive version of MPC, Generalized Predictive Control (Clarke et al. 1987), has also received considerable attention and been applied in industry.

In MPC the on-line control calculations consist of solving a linear or quadratic programming problem at each sampling instant, as new measurements become available. Although these calculations are complex and time consuming, the sampling periods are long enough (e.g. 5-30 minutes) so that the calculations can be performed on process control computers. An advantage of the MPC approach is that the loss of a sensor or an actuator can be accommodated by merely changing the corresponding inequality constraints. The dynamic process models are usually linear, empirical models 
in the form of difference equations which relate input and output variables. Experience has indicated that the model identification is a key step in the successful implementation of MPC techniques. Typically, the model is developed from a series of open-loop experimental tests. Additional information concerning the theory and application of MPC is available in recent survey articles (Garcia et al. 1989, Ricker 1991, Richalet 1993).

It is noteworthy that while MPC techniques such as DMC have been widely used in oil refineries, relatively few applications have been reported in other process industries such as pulp and paper (Dumont 1986). This omission is somewhat surprising because MPC is a general technique that does not rely on specific attributes of refining processes.

\subsection{Cross direction control of paper machines}

There is a second class of MIMO model-based control technique which has had widespread application in the pulp and paper industry. Several vendors market software packages which provide 'cross direction control (CDC)' of paper machines. The control objective is to minimize the variations of paper thickness, moisture content etc. across the width of the moving sheet of paper. The control strategy is based on an empirical MIMO model whose dynamics are dominated by a large time delay.

A 1985 article (Pulp \& Paper, February, 1985) reported that 960 CDC systems had already been installed at a cost of over $\$ 500$ million. The typical payout time was six months. Since the 960 systems represented $20 \%$ of the total number of paper machine computer systems, it is clear that this model-based control strategy received rapid acceptance. The CDC strategy is so widespread that virtually every new paper machine has at least one CDC application as part of its control system.

\section{Adaptive control}

During the past 20 years, adaptive control could well be the advanced control technique which has received the most attention. The basis concept is very appealing, namely, to have a control system that can automatically adjust its setting to accommodate changing process conditions. Thus the controller 'adapts' to changes in the process and to other unforeseen conditions. Detailed descriptions are available in textbooks (Åström and Wittenmark 1989, Hang et al. 1993), a survey of process control application (Seborg et al. 1986), and their respective bibliographies.

Although a variety of adaptive control strategies have been proposed in the control literature, the one that has received the most attention for process control applications is self-tuning control (STC). In STC a dynamic model of the process is updated on-line as conditions change. Then new values of the controller settings are calculated automatically based on the updated model parameters. Typically, the process models are empirical linear models in the form of difference equations, and recursive least squares techniques are used to update the model parameters.

Although the basic STC concept is quite simple, the dynamic behaviour of the resulting closed-loop system can be complex and difficult to analyse due to it nonlinear, time-varying nature. Furthermore, the accuracy of the identified model depends on the amount of 'excitation' that the process experiences. Hundreds of successful experimental applications of STC in industry and academia have been reported in the control literature. However, many of these applications can be classified as 
demonstrations which required specialists trained specifically in STC to design and implement the adaptive control systems.

Commercial adaptive control systems have been available since the early 1980's (Åström 1989). Probably the most widely used STC is the NOVATUNE controller which was originally marketed by ASEA in Sweden about 1982. A different type of adaptive controller, the EXACT controller, was introduced by the Foxboro Company in 1984. The EXACT is an adaptive PID controller which is based on a proprietary pattern recognition approach.

It is difficult to make accurate assessments of industrial utilization of adaptive control methods. Åström (1989) has reported that there were 100000 adaptive control loops running in 1988 but this estimate includes 'auto-tuners' which provide PID controller tuning on a one-time (on-demand) basis (Åström and Hägglund 1988). $\mathrm{He}$ also estimates that 2500 control loops were controlled by NOVATUNE controllers in 1988. An informal Foxboro estimate indicated that their EXACT controller was available in 15000 to 20000 control loops in 1987, as either a stand-alone controller or as part of Foxboro distributed control systems (Bristol 1987). However, the extent of current use is not known.

Adaptive control is a powerful concept that can produce significant improvements. It is important to note that it can also be implemented as a custom version of a specific controller, rather than as an application of a general purpose adaptive controller. For example, in a recent application of a nonlinear control strategy to a pH neutralization process, an adaptive version had to be employed in order to obtain satisfactory control (Henson and Seborg 1994).

\section{Nonlinear control techniques}

Because many important process control problems exhibit inherently nonlinear behaviour, conventional PID controllers and advanced controllers based on linear models (e.g. MPC) must be tuned conservatively to ensure stability over the entire range of operating conditions. This approach can result in very sluggish control for much of the operating region. Consequently, practical incentives exist for the development of control strategies based on nonlinear process models, especially if physically-based models could be used. This is an area of active research as indicated by a flurry of recent review papers (Kravaris and Kantor 1990, McLellan et al. 1990, Bequette 1991, Biegler and Rawlings 1991, Henson and Seborg 1991a).

Early nonlinear strategies tended to be specific to a particular problem (e.g. $\mathrm{pH}$ control) or rely on 'gain scheduling', a pre-programmed approach where the controller settings are varied according to known (or measured) changes in the process (Seborg et al. 1989). In recent years, general model-based control strategies have been developed using both physical models and a wide range of empirical NARMAX models which include neural networks and Hammerstein models. Analytical controller design methods have been developed for classes of nonlinear models based on an 'exact linearization' or 'different geometric' approach. By using an inverse of the process model (if it exists) in the control system, the closed-loop system will theoretically exhibit nominal stability (Kravaris and Kantor 1990, McLellan et al. 1990, Henson and Seborg 1991a). A nonlinear version of IMC (Henson and Seborg 1991b), Nonlinear Decoupling (Balchen 1991), and Generic Model Control (Lee 1991) can be regarded as special cases of this general approach. Generic Model Control has been applied in a variety of industrial applications. 
Nonlinear controllers can also be designed by extending the popular MPC approach for linear models to the more general situation where the process model is nonlinear (Biegler and Rawlings 1991). In this case, a nonlinear programming problem must be solved on-line at each sampling period instead of the much easier linear or quadratic programming problem in the MPC formulation.

Although the potential advantage of nonlinear control strategies are readily apparent, there are a number of significant difficulties which include:

1. Theoretical analysis of closed-loop performance properties such as stability and robustness is very difficult due to the nonlinear models. Thus a case study approach is often employed with no guarantee that the reported results can be generalized, or are even representative.

2. If an NLP problem is to be solved on-line at each sampling instant, the computational requirements can be substantial and convergence of the optimization algorithm difficult to guarantee.

Because nonlinear control strategies are still in an early state of development and there has only been a small number of practical applications, it is difficult to predict whether they will eventually have a significant impact on industrial practice.

\section{Artificial intelligence techniques}

In recent years there has been intense interest in developing artificial intelligence (AI) techniques for a wide variety of scientific and engineering applications. A comprehensive survey paper (Stephanopoulos and Han 1994) provides a thorough review of intelligent systems in process engineering and contains 385 references. The process control research in this area has largely been concerned with three AI methods: knowledge-based systems, neural networks, and fuzzy logic.

\subsection{Knowledge-based systems}

Knowledge-based systems (KBS), also referred to as expert systems, use a set of 'rules' to perform logical inferences about the state of a process operation or some other activity of interest. An early and highly visible demonstration project, the FALCON project, was a collaborative effort between Du Pont, Foxboro, and the University of Delaware during the period, 1983-1987 (Rowan 1992). The objective was to develop and apply knowledge-based methods for fault diagnosis in a full-scale chemical plant, an adipic acid converter. Although this pioneering project was judged to be only a partial success, it paved the way for many future Du Pont KBS applications.

Stephanopoulos and Han (1994) note that industrial applications of KBS systems have largely been concerned with either diagnostic and monitoring activities or supervisory control. Supervisory control applications have included the following problems: complex control schemes; recovery from extreme conditions; and emergency shutdowns. Stephanopoulos and Han (1994) also describe a number of industrial KBS applications.

A recent trade journal article by Samdani and Fouhy (1992) provides an overview of KBS applications in the process industries. They report that '... Du Pont has well quantified the benefits it is reaping from the thousand or so KBS's it has in place'. In the same issue, Samdani (1992) report that, '... [Du Pont] says that there are about 20000 more areas of applications yet to be tapped. For every dollar spent in implementing a KBS for process control, the payoff is from six to ten dollars per year'. 
Future applications of expert systems will be facilitated by real-time KBS which enable the user to integrate plant data and process models in an expert system shell which has a sophisticated graphical interface. This combination provides a powerful vehicle for on-line process monitoring, especially diagnostics and fault detection. At the present time, the most widely used system in the G2 product from the GENSYM Corporation (Cambridge, MA). It has been reported that over $1000 \mathrm{G} 2$ systems have been installed worldwide (Samdani and Fouhy 1992). In a recent application at a Monsanto-Krummich plant in Illinois, a G2 system provided the framework for a sensor and control loop validation system for over 600 measurement points. The diagnostic system was able to successfully identify a variety of actual faults and is being modified for use in other plants (Thompson and Mertz 1993).

The early enthusiasm for KBS has been tempered by the realization that a considerable effort is required to codify the available expertise. Furthermore, if each potential application has a significant number of unique features, it is less feasible to spread the development costs over a large number of projects. Despite this inherent problem, the industrial employment of KBS for applications such as process diagnosis and supervisory control is significant and growing at an impressive rate (Stephanopoulos and Han 1994).

\subsection{Neural networks}

Neural networks provide a powerful approach for developing empirical nonlinear models for a wide variety of physical phenomena. In the area of process control, they have been used for a variety of traditional activities, such as developing nonlinear dynamic models and control system design (Bhat and McAvoy 1990, Hunt et al. 1992). Neural networks also provide a promising approach for pattern recognition problems such as sensor data analysis and fault detection where traditional modelling techniques are not easily applied.

Standard neural network models consist of three layer networks with sigmoidal functions used as the 'activation function' for each neuron in the hidden layer. However, networks which consist of linear combinations of radial basis functions offer significant theoretical and computational advantages over the standard neural networks (Chen et al. 1990, Leonard and Kramer 1991, Pottmann and Seborg 1992a, b, Stephanopoulos and Han 1994). Furthermore, a priori physical information such as known steady-state relations and some types of constraints can easily be incorporated into the otherwise empirical models (Pottmann and Seborg 1992b).

The commercial availability of neural network software for use by non-specialists should continue the current widespread interest in neural network applications for process control. However, at the present time it is difficult to assess the extent to which process control applications of neural networks are being used in industry.

\subsection{Fuzzy control systems}

Fuzzy logic provides a conceptual framework for practical problems where some process variables are represented as "linguistic variables" which have only a few possible values (e.g. very large, large, normal, small etc.). The linguistic variables can then be processed using a set of rules. Thus applications of fuzzy logic and fuzzy control can be viewed as special cases of KBS which have fuzzy boundaries for the rules.

Unlike more general KBS and neural nets, fuzzy control strategies have appeared in the control literature for over 20 years. Early process control applications consisted 
of demonstrations that fuzzy control could be used to control simple laboratory apparatus. In recent years, the success of fuzzy control in Japan, especially in consumer products such as washing machines and camcorders, has generated a new wave of interest. Industrial applications of fuzzy control to process control problems have begun to appear more frequently in Japan and Europe than in the U.S. But even in Japan, a survey has indicated that MPC has been more widely used in the process industries than any of the three AI techniques considered in this section (Yamamoto and Hashimoto 1991).

There has been considerable controversy concerning fuzzy controllers and their relative merits viz. conventional control and model-based control. One of the reasons for this controversy is that there is no theoretical framework for analysing the closed-loop properties of fuzzy control systems. As Stephanopoulos and Han (1994) aptly note, 'Using fuzzy controllers takes a lot of testing and/or faith.'

A recent trade journal article provides an overview of fuzzy logic applications in the U.S. (Samdani et al. 1993). It states, 'Although FL has yet to prove its worth to most [process] engineers, particularly in the U.S., vendors believe that it is here to stay, and are steadily commercializing FL-based products.' They report that more than 10000 FL-embedded PIC controllers have been sold by the Yokogawa Corp. of America. These controllers use FL for auto-tuning. On the other hand, a recent survey of FL applications in engineering cited only a few control applications outside of Japan (Dubois 1993). Stephanopoulos and Han (1994) describe a number of industrial applications of fuzzy control, primarily at the supervisory control level.

Regrettably, a high degree of 'hype' was associated with the initial introduction of these three AI technologies and consequently, early expectations were not always fulfilled. But it is important to keep in mind that these are new approaches for process control and that software still tends to be 'first generation'. As the technology and available software continues to improve, widespread industrial applications are quite likely. Also, the individual AI techniques can be combined to good advantage, for example, by embedding neural networks and fuzzy logic in knowledge-based systems. In particular, neural networks have been proposed for the preliminary screening of data that are analysed further by expert systems in diagnostic and monitoring applications.

\section{Process monitoring and on-line diagnostics}

The complexity of many processes and the increasing degree of data acquisition has resulted in an overwhelming amount of information that is available to the operating personnel. Consequently, there are considerable practical incentives for the development of diagnostic techniques that can be used to monitor the performance of both industrial plants and their control systems.

The traditional approach for monitoring processes has been to perform simple limit checking on the magnitudes or rates of change of individual measurements to ensure that they are within acceptable limits. Due to the keen emphasis on quality and quality control in the 1980s, the traditional limit checking approach has been augmented in some of the process industries by the widespread use of statistical quality control (SQC) charts such as Shewhart and CUSUM charts (Wadsworth et al. 1986, MacGregor 1988).

By itself, this type of SQC application neither determines the reason for the process being 'out of a state of statistical control' nor does it automatically determine 
a corrective action to bring the process back to the desired (target) condition. Nevertheless, SQC has enjoyed widespread application and does provide valuable information about the state of the process, especially when the data are noisy and/or are available on only an infrequent basis. SQC has been widely used in the semiconductor processing industry and in some portions of the chemical industry. For example, a 1988 article reports the Du Pont has more than 10000 CUSUM charts being actively used (MacGregor 1988). In contrast, SQC does not appear to be widely used in other process industries such as oil refining and pulp and paper.

In recent years, the development of improved techniques for on-line monitoring and fault detection has developed into a major research area, both in process control and in the more general control engineering community. An overview of the rapidly developing field is provided by a recent conference (Dhurjati 1991), book (Patton et al. 1989) and survey article (Frank 1990). Typical approaches for fault detection include model-based methods (e.g. parameter estimation, residual evaluation), statistically based techniques, and the AI techniques described in the previous section.

Stephanopoulos and Han (1994) provide an excellent overview of the use of AI techniques for a wide variety of process monitoring activities. Their paper describes a number of industrial applications and cites many survey papers for this fast growing field. Among the statistically-based diagnostic techniques, multivariate techniques such as Principal Component Analysis (PCA) and Projection to Latent Structures (PLS) provide powerful alternatives to traditional single variable SQC charting methods (MacGregor 1994). They require a good database of past operation but little else in the way of a priori information.

In addition to monitoring the performance of the process, it is very desirable to know how well the control system is performing. This would appear to be an important issue for both standard PID control systems and model-based control strategies such as MPC. As Benson (1994) has recently noted, 'If as a process industry we do not measure our control performance, maybe it is not surprising that it does not improve'. Some key issues in control system performance monitoring are:

1. Is the control system performing up to expectations?

2. If not, can the performance be significantly improved using the present instrumentation and control configuration? (e.g. by sensor or actuator maintenance or by re-tuning the controller.)

3. If the answer to Question 2 is negative, can significant improvement be obtained by application of an 'advanced' control strategy?

Despite the importance and centrality of these issues in process control, they have received little attention until recently when a few papers have started to appear (Shinskey 1990, Åstrōm et al. 1992, Desborough and Harris 1993, Hägglund 1994, Miao and Seborg 1994).

\section{Conclusions}

Process control continues to be an intriguing field with important theoretical problems and challenging applications. Most advanced control strategies continue to be model-based but AI-based techniques are receiving widespread attention. A key research issue is how to integrate AI-based strategies with model-based and statistical approaches in a practical and cost-effective manner. 


\section{REFERENCES}

ÅsTRÖM, K.H. (1989). Toward intelligent control. IEEE Control Systems Magazine, 9(4), 60-64. ÅströM, K. J. and HäGGLund, T. (1988). Automatic Tuning of PID Controllers (Instrument Soc. of America, Research Triangle Park, NC).

Åström, K. J., HANG, C. C., Persson, P. and Ho, W. K. (1992). Towards intelligent PID control. Automatica, 28, 1-9.

ÅströM, K. J. and WrTtenMARK, B. (1989). Adaptive Control (Addison Wesley, Reading, MA). BALCHEN, J. G. (1991). Nonlinear decoupling in process control. Model. Indent. Control, 2, 81-94.

Balchen, J. G. and Mummé, K. (1988). Process Control: Structures and Applications (Van Nostrand Reinhold, NY).

BequeTte, B. W. (1991). Nonlinear control of chemical processes-a review. Ind. Eng. Chem. Res., 30, 1391-1413.

BENSON, R. S. (1994). The process industry requirements of advanced control techniques: challenges and opportunities. Preprints of the IFAC ADCHEM '94 Symposium, 215-224, Kyoto, Japan, May, 1994.

BHAT, N. V. and MCAvOY, T. J. (1990). Use of neural nets for dynamic modelling and control of chemical process systems. Computers \& Chem. Eng., 14, 573-582.

BIEGLER, L. T. and RAWLINGS, J. B. (1991). Optimization approaches to model predictive control. Proc. Chemical Process Control IV, Y. Arkun and W. H. Ray (editors) (AIChE, NY).

BRISTOL, E. H. (1987). private communication.

Chen, S., Billings, S. A., Cowan, C. F. N. and Grant, P. M. (1990). Practical identification of NARMAX models using radial basis functions. Int. J. Control, 52, 1327-1350.

Clarke, D. W., MohTADI, C. and TufTS, P. S. (1987). Generalized predictive control-Part I. The basic algorithm. Automatica, 23, 137-148.

Desborough, L. and Harris, T. (1993). Performance assessment measures for univariate feedforward/feedback control. Can. J. Chem. Eng., 71, 605-616.

Dhurjati, P. S. (editor) (1992). Proc. IFAC Symposium on On-Line Fault Detection and Supervision in the Chemical Process Industries, Newark, DE (Pergamon, NY).

DuboIs, D. (1993). Fuzzy sets: A survey of engineering applications. Comput. \& Chem. Eng., 17, S373-380.

DumONT, G. (1986). Application of advanced control methods in the pulp and paper industry-a survey. Automatica, 22, 143-154.

Fisher, D. G. (1991). Process control: an overview and personal perspective. Can. J. Chem. Eng., 69, 5-26.

Fisher, D. G. and SEborg, D. E. (1976). Multivariable Computer Control: A Case Study. (Elsevier, NY).

FRANK, P. M. (1990). Fault diagnosis in dynamic systems using analytical and knowledge-based redundancy - a survey and some new results. Automatica, 26, 459-474.

GARCIA, C. E. and MORARI, M. (1982). Internal model control 1. A unifying review and some new results. Ind. Eng. Chem. Des. \& Dev., 21, 308-322.

Garcia, C. E., PretT, D. M. and Morari, M. (1989). Model predictive control: theory and practice- a survey. Automatica, 25, 335-348.

Gusciora, P. H., MCAMiS, J.H., SORENSEN, R. C. and CuTLER, C. R. (1992). Experiences applying DMC on a Model IV FCC. Paper presented at the AIChE Annual Mtg (Miami Beach, FL), Nov., 1992.

HÄGGLund, T. (1994). Automatic monitoring of control loop performance. Conf. on Control Systems in the Pulp and Paper Industry (Control Systems '94). Stockholm.

Hang, C. C., LeE, T. H. and Ho, W. K. (1993). Adaptive Control (Instrument Soc. of America, Research Triangle Park, NC).

Henson, M. A. and SEBORG, D. E. (1991a). A critique of differential geometric strategies for process control. J. Process Control, 1, 122-139.

Henson, M. A. and SEBorG, D. E. (1991b). An internal model control strategy for nonlinear systems. AIChE J., 37, 1065-1081.

Henson, M. A. and SeborG, D. E. (1994). Adaptive nonlinear control of a pH neutralization process. IEEE Trans. Control Systems \& Technology (in press).

Hunt, K. J., Sbarbaro, D., Zbikowski, R. and Gawthrop, P. J. (1992). Neural networks for control systems-a survey. Automatica, 28, 1083-1112. 
Kravaris, C. and Kantor, J. C. (1990). Geometric methods for nonlinear process control: 1 . Background. Ind. Eng. Chem. Res., 29, 2295-2310.

McLellan, P. J., Harris, T. J. and BACON, D. W. (1990). Error trajectory descriptions of nonlinear controller designs. Chem. Eng. Sci., 45, 3017-3034.

LEE, P. L. (1991). Direct use of nonlinear models for process control. Proc. Chemical Process Control IV, Y. Arkun and W. H. Ray (editors) (AIChE, NY).

LEONARD, J. A. and KRAMER, M. A. (1991). Radial basis function networks for classifying process faults. IEEE Control Systems Mag., 11 (3), 31-38.

MaCGregor, J. F. (1988). On-line statistical process control. Chem. Eng. Progr., 84 (10), 21-31.

MACGREGOR, J. F. (1994). Statistical process control of multivariable processes. Preprints of the IFAC ADCHEM '94 Symposium, 215-224, Kyoto, Japan, May, 1994.

Miao, T. and Seborg, D. E. (1994). Paper to be presented at the AIChE Annual Mtg., San Francisco, Nov., 1994.

MORARI, M. (1994). Current directions in process control theory. Preprints of the IFAC ADCHEM '94 Symposium, 849-863, Kyoto, Japan, May, 1994.

Muske, K., YounG, J., Grosdidier, P. and TANI, S. (1991). Crude unit product quality control. Computers in Chem. Eng., 15, 629-638.

Patton, R., Frank, P. and Clark, R. (editors), (1989). Fault Diagnosis in Dynamic Systems (Prentice Hall, NY).

Pottmann, M. and SEBorg, D. E. (1992a). Identification of nonlinear processes using reciprocal multiquadric functions. J. Process Control, 2, 189-203.

POTTMANN, M. and SEBORG, D. E. (1992b). Identification of nonlinear processes incorporating a prori physical knowledge. Paper presented at the 1992 AIChE Annual Mtg (Miami Beach, FL), Nov., 1992.

RichAlET, J. (1993). Industrial applications of model based predictive control. Automatica, 29, 1251-1274.

Ricker, N. L. (1991). Model predictive control: state of the art. in Chemical Process Control-CPC IV, Y. Arkun and W. H. Ray (editors) (AIChE, NY).

RIJNSDORP, J.E. and SEBORG, D. E. (1976). A survey of experimental applications of multivariable control to process control problems. AIChE Symposium Series, 72, (159), 112-123.

ROWAN, D. A. (1992). Beyond FALCON: industrial applications of knowledge-based systems. in Proc. IFAC Symposium on On-Line Fault Detection and Supervision in the Chemical Process Industries, P. S Dhurjati, (editor) (Newark, DE).

SAMDANI, G. (1992). Managing knowledge. Chem. Eng., 99 (4), 5.

SAMDANI, G. and FouHy, K. (1992). Smart software. Chem. Eng., 99 (4), 30-33.

SAMDANI, G., FouHY, K. and MoOre, S. (1993). Fuzzy logic: more than a play on words. Chem. Eng., 100 (2), 30-33.

Seborg, D. E., Edgar, T. F. and Mellichamp, D. A. (1989) Process Dynamics and Control (John Wiley \& Sons, NY).

Seborg, D. E., Shah, S. L. and Edgar, T. F. (1986). Adaptive control strategies for process control: a survey. AIChE J., 32, 881-913.

SHINSKEY, F. G. (1990). How good are our controllers in absolute performance and robustness? Measurement and Control, 23 (5), 114-121.

StePhanOPOUlos, G. and Han, C. (1994). Intelligent systems in process engineering: a review. Preprints of the 5th Internat. Symposium on Process Systems Eng. (PSE 94), 1339-1366, Kyongju, Korea, June, 1994.

Thompson, L. and Mertz, G. (1993). Real-time expert system implementation at Monsanto-Krummrich. Automatica, 29, 1177-1183.

WAdSWORTH, H. M., STEPHENS, K. S. and GodFreY, A. B. (1986). Modern Methods for Quality Control and Improvement (John Wiley, NY).

ZumWALT, R. E. and WolfaNG, G. (1980). Directions in computer control research and practice. Proc. of the 1980 Joint Automatic Control Conf., San Francisco, paper TA5-A.

Y АмАмOTO, S. and HASHIMOTO, I. (1991). The view from Japanese industry. in Chemical Process Control-CPC IV, Y. Arkun and W. H. Ray (editors) (AIChE, NY). 